TITLE:

\title{
The Finite Beta Effects on the Toroidal Field Ripple in a Tokamak Plasma
}

\section{$\operatorname{AUTHOR}(S):$}

Bunno, M.; Nakamura, Y.; Suzuki, Y.; Shinohara, K.; Matsunaga, G.; Tani, K.

\section{CITATION:}

Bunno, M. ... [et al]. The Finite Beta Effects on the Toroidal Field Ripple in a Tokamak Plasma. Plasma Science and Technology 2013, 15(2): 115118

ISSUE DATE:

2013-02

URL:

http://hdl.handle.net/2433/173038

\section{RIGHT:}

C 2013 IOP Publishing.; この論文は出版社版でありません。引用の際 には出版社版をご確認ご利用ください。; This is not the published version. Please cite only the published version. 


\title{
The finite beta effects on the toroidal field ripple in a tokamak plasma
}

\author{
${ }^{\text {a) }}$ M.Bunno, ${ }^{\text {a) }}$ Y.Nakamura, ${ }^{\text {b) }}$ Y.Suzuki, ${ }^{\text {c) }}$ K.Shinohara, ${ }^{\text {c) }}$ G.Matsunaga and ${ }^{\text {d) }}$ K.Tani \\ a) Graduate School of Energy Science, Kyoto University, Gokasho, Uji 611-0011, Japan \\ ${ }^{b)}$ National Institute for Fusion Science, Toki, Gifu 509-5292, Japan \\ c) Japan Atomic Energy Agency, Naka, Ibaraki 311-0193, Japan \\ ${ }^{d)}$ Nippon Advanced Technology Co., Ltd, Naka, Ibaraki 311-0102, Japan \\ bunno.michinao.44v@st.kyoto-u.ac.jp
}

Keywords: ripple, VMEC, ITER, alpha particle, F3D-OFMC

PACS numbers: 52.20.Dq, 52.55.Dy, 52.55.Fa, 52.65.Cc, 52.65.Kj

\begin{abstract}
The efficiency of the energetic ion confinement is reduced in a tokamak plasma by the non-axisymmetric field, namely the ripple field. The ripple field is produced by the finite number of the toroidal field coils. It is affected by the non-axisymmetric finite beta effect. The three-dimensional MHD equilibrium calculation code VMEC is used to analyze the non-axisymmetric finite beta effect in a ripple tokamak. In the VMEC code, the flux coordinates are used, so the calculation region is limited to the area of plasma. To calculate the orbit of them outside the plasma, we develop the field calculation code, which is based on the Biot-Savart law. The details of the method and results are described in this study.
\end{abstract}

\section{Introduction}

A tokamak plasma is often approximately treated as an axisymmetric system. However, the finite number of the toroidal field coils breaks the axisymmetry of the plasma. The non-axisymmetric magnetic fields along the toroidal angle $\varphi$ are called toroidal field (TF) ripples and the amount of energetic ion losses is increased by them. These losses produce several serious problems in a tokamak, such as the deterioration of the plasma heating efficiency and the concentration of heat loads on the first wall.

To analyze these problems, the accurate magnetic field structures including the finite beta effects are necessary because the plasma equilibrium current also changes the magnetic field configurations. In the previous studies about energetic ion losses, the two-dimensional (2D) MHD equilibrium equations have been usually used by assuming the plasma to be axisymmetric in a tokamak plasma. The resulting MHD equilibrium field is axisymmetric. However, the three-dimensional (3D) magnetic field can be approximately obtained by superimposing the non-axisymmetric components of the vacuum field. The VMEC code is one of the 3D, free boundary MHD equilibrium calculation code and it has been ordinary used in a helical plasma ${ }^{[1][2]}$. These days, the VMEC code is often used to analyze the energetic ion losses also in a tokamak ${ }^{[3]-[5]}$. The full 3D MHD equilibria can be easily obtained by the VMEC code, while the calculation region is limited to the inside plasma region because flux coordinates are used in this code. In the result, the fraction of the lost energy and the loss position of the energetic ions could not be accurately evaluated.

The equilibrium field is described as the sum of the magnetic fields from the external coil current and the plasma equilibrium current. If the plasma is assumed to be a kind of coils, the equilibrium field can be calculated from every coil shape and every coil current by using the Biot-Savart law. We developed the equilibrium field calculation code, which is based on this idea. The separatrix structures and the heat loads on the wall can be analyzed with this code.

In Sec.2, the field calculation code is precisely explained. In Sec.3, the 3D magnetic configuration, which is obtained by this code, is analyzed. Finally, the summaries and conclusions are described in Sec.4.

\section{Details of the developed calculation code}

The equilibrium field can be calculated inside the plasma by the VMEC code in the flux coordinates $(s, \theta, \zeta)$, where $s, \theta$ and $\zeta$ are the normalized toroidal magnetic flux, the poloidal angle and the toroidal angle, respectively. The toroidal angle $\varphi$ of the cylindrical coordinates $(R, Z, \varphi)$ is used as the toroidal angle $\zeta$ of the flux coordinates.

Inside the plasma, the field in the flux coordinates is transformed to that in the cylindrical coordinates by the inverse mapping method ${ }^{[6]}$. However, outside the plasma boundary, this inverse mapping method can not be used. As previously mentioned, the 

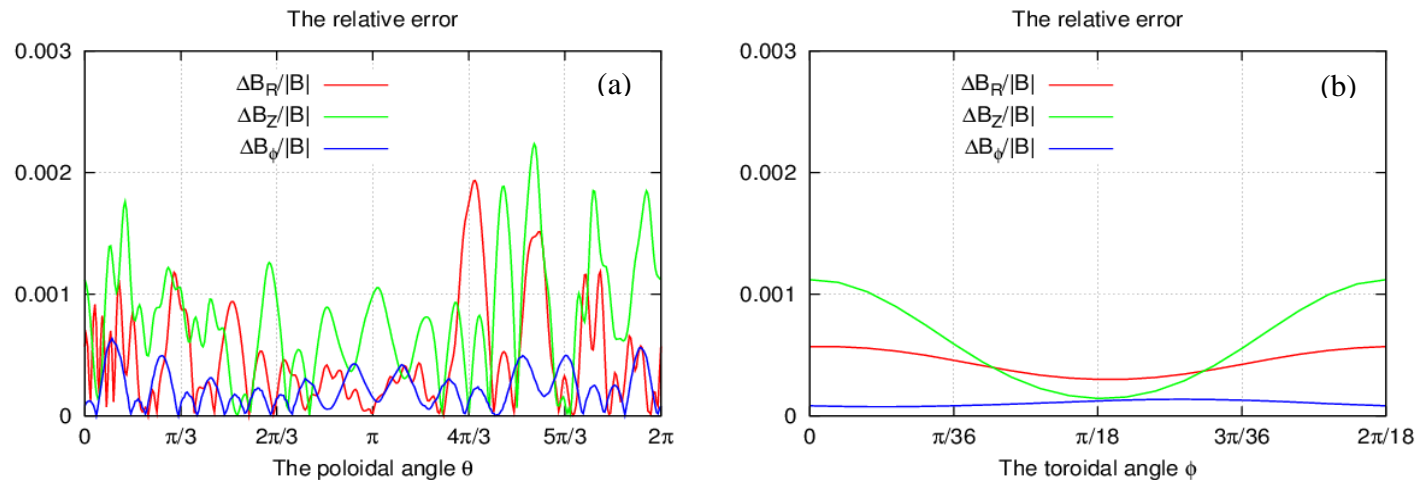

Fig.1 The relative error of each field component between the results from the VMEC code and the developed field calculation code on the plasma boundary (a) along the poloidal angle $\theta$ at $\varphi=0$ and

(b) along the toroidal angle $\varphi$ at $\theta=0$

equilibrium field can be calculated outside the plasma by the Biot-Savart law,

$$
\vec{B}(\vec{r})=\vec{B}_{\text {ext }}(\vec{r})+\int \frac{\mu_{0} \vec{j}_{p}\left(\overrightarrow{r^{\prime}}\right) \times\left(\vec{r}-\vec{r}^{\prime}\right)}{4 \pi\left|\vec{r}-\vec{r}^{\prime}\right|^{3}} d V,
$$

where $j_{p}$ is the plasma equilibrium current and $B_{\text {ext }}$ is the magnetic field from the external coil currents.

We compare magnetic fields which are obtained by both calculation methods on the plasma boundary. The calculation model is referred to as the steady state 9MA scenario in ITER ${ }^{[4][6]}$. Figure 1 (a) and (b) show the relative error of each field component $\Delta B_{R} \backslash B\left|, \Delta B_{Z}\right| B \mid$ and $\Delta B_{\varphi} \backslash B \mid$ on the plasma boundary between the results from the VMEC code and the developed field calculation code along the poloidal angle $\theta$ at $\varphi=0$ and along the toroidal angle $\varphi$ at $\theta=0$, respectively. Because of $1 /|r-r|$, the calculation error appear in these figures, but the maximum relative error is only about $0.2 \%$. Therefore, we can conclude that the developed field calculation code has high enough numerical accuracy. In this case, the normalized toroidal magnetic flux $s$, the poloidal angle $\theta$ and the toroidal angle $\zeta$ is divided to 40,360 and 720 , respectively. To calculate the field structures, a lot of computational time and resources are required. Therefore, the equilibrium field is transformed from the flux coordinates to the cylindrical coordinates inside the plasma and calculated by the Biot-Savart law outside the plasma.

\section{Results}

\subsection{Orbit of fusion alpha particles outside the plasma}

In this study, the steady state 9MA scenario in ITER is used as the calculation model. Figure 2 (a) and (b) show the safety factor profile $q$ and the pressure profile $\mathrm{P}[\mathrm{MPa}]$ as the function of the normalized toroidal magnetic flux s. The MHD equilibria are calculated by the VMEC code. The equilibrium field is transformed from the flux coordinates to the cylindrical coordinate by the calculation method explained in Chapter 2. The black lines in Fig.2 (c) show the poloidal magnetic flux surfaces. The separatrix configuration can be clearly seen in Fig.2 (c).
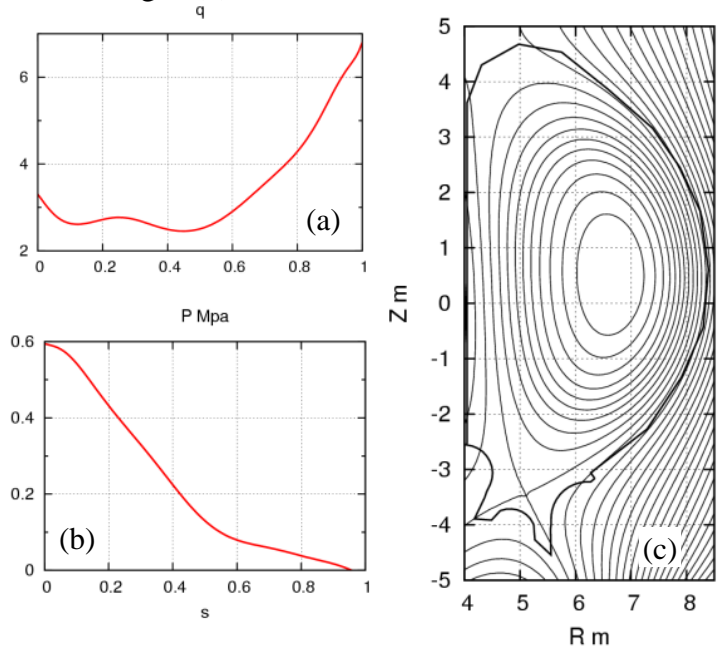

Fig.2 (a) The safety factor profile q, (b) the pressure profile $\mathrm{P}[\mathrm{MPa}]$ and (c) the poloidal magnetic flux surfaces in the steady state 9MA scenario in ITER

First, the orbits of the fusion alpha particles are calculated by solving the guiding center orbit equation in the cyrindlical coordinates. In this calculation, the collision is ignored. Therefore, if the guiding center reaches the boundary (the plasma boundary or the first wall) the particles are assumed to be loss, else if the tracing time exceeds $0.01 \mathrm{~s}$, they are assumed to become non-loss particle. The green dots in Fig.3 (a) show the birth points of 10,000 fusion alpha particles on the poloidal cross-section. In the case of the VMEC code alone, the orbit can be traced only inside the plasma. However, thanks to the additional procedure, we can calculate it up to the first wall.

In Fig.3 (a), the blue dots show the position of the particles, which reach the plasma boundary, and the the red dots show the position of the particles, which reach the first wall. These positions are quite different 

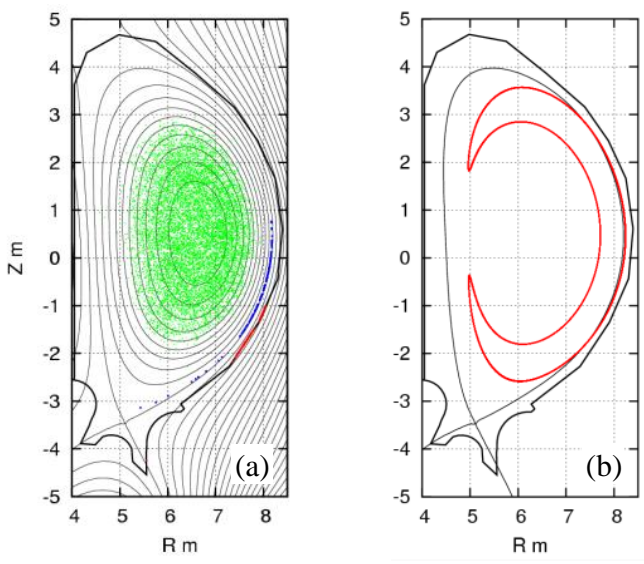

Fig.3 (a) The birth points of the fusion alpha particles (the green dots), the positions of the loss particles on the plasma boundary (the blue dots) and these positions on the first wall (the red dots), and (b) the orbit of banana particle which reaches the plasma boundary

between the two results, so the heat loads can not be correctly evaluated only from the VMEC results. All particles, which reach the plasma boundary, have often been assumed to be loss because the magnetic field line is not closed outside the plasma. In this calculation, 124 of the total 10,000 fusion alpha particles reach the plasma boundary. However, only 98 of these collide with the first wall. This difference mainly comes from the width of the banana orbit. Figure 3 (b) shows the orbit of a particle which has a large banana width. It only reaches the plasma boundary during part of its orbit, but does not become loss. It clearly shows that the loss could not be correctly dtermined only from the VMEC results, so the additional procedure in this study is required.

\subsection{Non-axisymmetric field from the plasma current}

The strongest advantage of the VMEC code is that are able to research the non-axisymmetric finite beta effect. We should compare the full 3D MHD equilibria and the approximated $3 \mathrm{D}$ field, which is obtained by superimposing the vacuum ripple field on the 2D MHD equilibrium field. The ripple ratio $\delta$ is defined by

$$
\delta=\frac{B_{\max }-B_{\min }}{B_{\max }+B_{\min }},
$$

where $B_{\max }$ and $B_{\min }$ are the maximum and minimum field strength along the toroidal direction $\varphi$ at the fixed $(R, Z)$ position $^{[4]}$. In this study, the $\delta$ of the full $3 \mathrm{D}$ VMEC and the 3D approximated field are called $\delta_{3 \mathrm{D}}$ and $\delta_{2 \mathrm{D}}$, respectively. Figure 4 (a) shows the $\delta_{3 \mathrm{D}}$ distribution on the poloidal cross-section. $\delta_{3 \mathrm{D}}$ is the strongest at the outer torus and the maximum value about 0.01 . The $\delta_{3 \mathrm{D}}-\delta_{2 \mathrm{D}}$ distribution on the poloidal cross-section is shown in Fig.4 (b) to show the non-axisymmetric finite beta effect on the $\delta$ distribution. It is obviously seen from Fig.4 (b) that
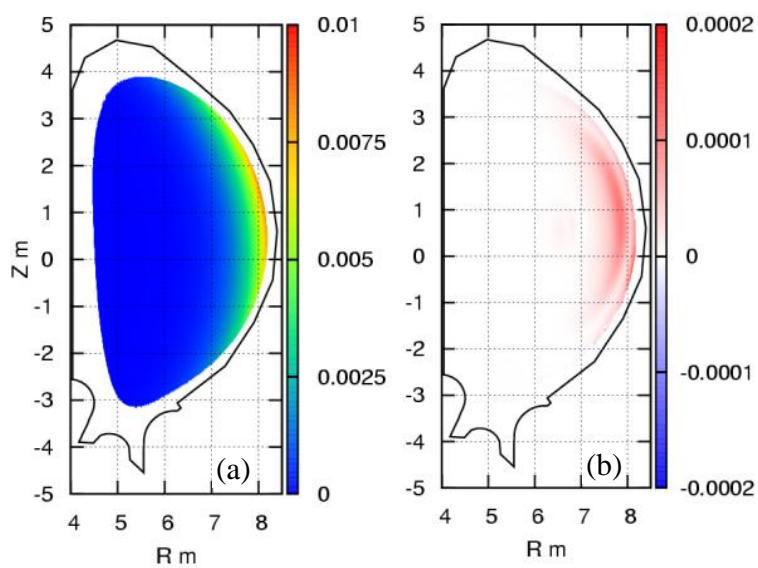

Fig.4 (a) The ripple ratio $\delta$ for the full 3D MHD equilibrium field $\left(\delta_{3 \mathrm{D}}\right)$ and (b) the diffecence of $\delta$ between the full 3D and the approximated $3 \mathrm{D}$ field $\left(\delta_{3 \mathrm{D}}-\delta_{2 \mathrm{D}}\right)$

the non-axisymmeric finite beta effect increase the ripple ratio. The maximum difference between $\delta_{3 \mathrm{D}}$ and $\delta_{2 \mathrm{D}}$ is about 0.0002 . We should analyze how this increase in $\delta$ affects on the energetic ion losses.

A more reliable orbit calculation code is required to obtain more realistic and accurate results. The F3D-OFMC code has been widely used to calculate the ripple induced orbit losses ${ }^{[7][8]}$. The guiding center orbits are traced by this code while considering the slowing down and the pitch angle scattering. In this code, the finite Larmor radius effect is considered near the first wall. Figure 5 shows the loss rate of 10,000 fusion alpha particles calculated by the F3D-OFMC code. The red and blue line show the loss rate of the full $3 \mathrm{D}$ VMEC results and the $3 \mathrm{D}$ approximated field, respectively. The loss rate is defined as the ratio of the total kinetic energy of all loss particles at the loss point and the total initial energy of all particles. The final loss rates for the full 3D VMEC and the 3D approximated field are about 0.040 and 0.039 , respectivity. The non-axisymmetric finite beta effect increases the ripple ratio and the loss rate by a small percentage. The red and blue points in Fig. 6 show the loss positions for the two results. The shape of the first wall and the finite number of the toroidal fied coils are also shown in this figure. The loss positions for both results are concentrated on the bottom of the ripple (between the toroidal field coils). A clear difference between them could not be seen on this figure. We can conclude that the non-axisymmetric finite beta effect is negligibly small and the approximated 3D field has high numerical accuracy under the conditions of this research. 


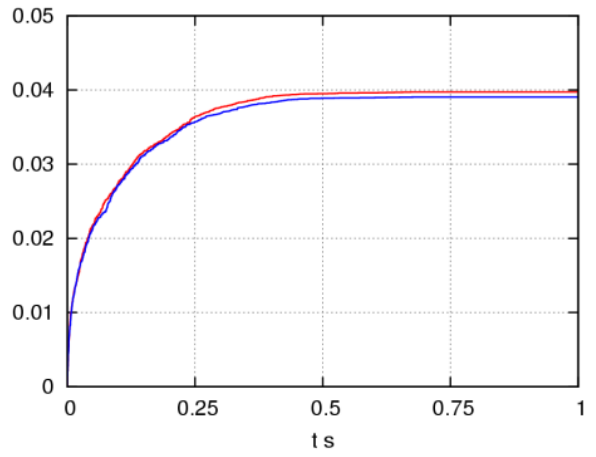

Fig.5 The loss late for the full 3D MHD field (the red line) and the 3D approximated field (the blue line)

\section{Summary}

In this study, the VMEC code is used to analyze the non-axisymmetric finite beta effect. However, the equilibrium field could not be calculated inside the plasma because the flux coordinates are used. We propose and develop the field calculation code, which is based on the Biot-Savart law. The magnetic field configuration, which obtained by this method, is compared to the VMEC result and the maximum relative error is less than $1 \%$.

Next, the orbits of the fusion alpha particles are calculated. If particles reach the plasma boundary, they are often assumed to be loss because the magnetic field line is not closed outside the plasma. However, some of them does not become loss even if they reach the plasma boundary during part of its orbit. In this study, 124 of the total 10,000 fusion alpha particles reach the plasma boundary and only 98 of these collide with the first wall. The loss could not be correctly dtermined only from the VMEC results. Therefore, the additional procedure in this study is required.

To analyze the non-axisymmetric finite beta effect on the ripple induced orbit losses, we compare the results of the full 3D MHD equilibria and the 3D approximated field, which is obtained by superimposing the vacuum ripple field on the 2D MHD equilibrium field. The non-axisymmetric finite beta effect increases the ripple ratio by a small percentage. The effect of this increase is investigated by tracing the orbit of the fusion alpha particles by the F3D-OFMC code. The loss rates of the full 3D MHD equilibria and 3D approximated field are 0.040 and 0.039 , respectively. It shows that the loss rate is also increased by the non-axisymmetric finite beta effect. However, this effect is negligibly small. Furthermore, a difference of the loss positions could not be clearly found between the two 3D fields. Therefore, we can conclude that the non-axisymmetric finite beta effect is not strong under the condition of this research.

An ITER plasma of the volume averaged beta $<\beta>\sim 2 \%$ is used as the calculation model in this study,

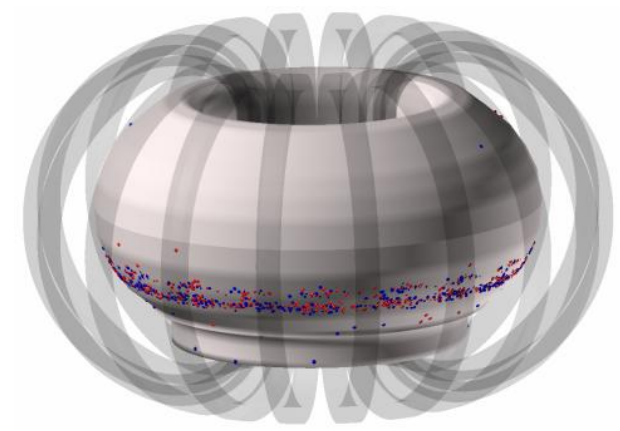

Fig.6 The loss position of the fusion alpha particles on the first wall for the full 3D MHD equilibria (the red dots) and the 3D approximed field (the blue dots)

which has small non-axisymmetric effect and small finite beta effects. In order to create a tokamak reactor with smaller ripple induced losses, it must be clarified how much do the number of the toroidal field coils, the aspect ratio and the beta value change the energetic ion losses.

\section{Acknowledgement}

The authors would like to thank S.P.Hirshman and D.A.Spong for providing VMEC code and ITER equilibrium data. This work is performed with the support and auspices of the NIFS Collaborative Research Program.

\section{References}

[1] S.P.Hirshman and H.K.Meier Phys. Fluids 28 (1985) 1387.

[2] S.P.Hirshman, W.I.van Rij and P.Merkel, Comput. Phys. Comm. 43 (1986) 143.

[3] Y.Suzuki, Y.Nakamura and K.Kondo, Nucl. Fusion 43 (2003) 406415.

[4] E.Strumberger, S.Günter, P.Merkel, E.Schwarz and C.Tichmann, Nucl. Fusion 50 (2010) 025008 .

[5] D.A.Spong, Physics of Plasmas 18 (2011) 056109.

[6] "S.E.Attenberger, W.A.Houlberg and S.P.Hirshman, Some Practical Considerations Involving Spectral Representations of 3D Plasma Equilibria, Tech. Rep. ORNL/TM-10412 (1987)

[7] K.Shinohara, T.Kurki-Suonio, D.Spong, G.Vlad, O.Asunta, K.Tani, E.Strumberger, S.Briguglio, T.Koskela, S.Günter, G.Karmer, S.Putvinski and K.Hamamatsu, Nucl. Fusion 51 (2011) 063028.

[8] K.Tani, M.Azumi, H.Kishimito and S.Tamura, J. Phys. Soc. Japan. 50 (1981) 1726 